\title{
Compensation for the Effect of Vacuum Chamber Eddy Current by Digital Signal Processing for Closed Orbit Feedback*
}

\author{
Y. Chung, L. Emery, and J. Kirchman \\ Argonne National Laboratory, Argonne, IL 60439
}

\begin{abstract}
The Advanced Photon Source (APS) will implement both global and local beam position feedback systems to stabilize the particle and $\mathrm{X}$-ray beams. The relatively thick (1/2") aluminum storage ring vacuum chamber at corrector magnet locations for the local feedback systems will induce significant eddy current. This will reduce the correction bandwidth and could potentially destabilize the feedback systems. This paper describes measurement of the effect of the eddy current induced in the APS storage ring vacuum chamber by a horizontal/vertical corrector magnet and its compensation using digital signal processing at $4 \mathrm{kHz}$ sampling frequency with proportional, integral, and derivative (PID) control algorithm for closed orbit feedback. A theory of digital feedback to obtain the linear system responses and the conditions for optimal control will also be presented. The magnet field in the vacuum chamber shows strong quadrupole and sextupole components varying with frequency, in addition to significant attenuation and phase shift with bandwidth $(-3$ dB) of $20 \mathrm{Izz}$ for horizontal and $4 \mathrm{~Hz}$ for vertical fields relative to the magnet current. Large changes in the magnet resistance and inductance were also observed, as the result of reduced total magnetic energy and increased Ohmic heat loss.
\end{abstract}

\section{INTRODUCTION}

In the APS storage ring, an extensive beam position correction system will be implemented comprised of many corrector magnets (318 total) and beam position monitors (BPMs) monitoring the positions of the positron and photon beams. The AC corrector magnets, which will correct beam motion of up to $100 \mu \mathrm{rad}$ at $25 \mathrm{~Hz}$, induce eddy current in the relatively thick (1/2") aluminum vacuum chamber of the storage ring for the local beam position feedback systems. This results in significant attenuation and phase shift of the magnet field even at frequencies as low as $5 \mathrm{~Hz}$. The global orbit feedback system uses a thin stainless steel chamber and is not significantly affected by the eddy current effect.

In order to avoid the problems characteristic of analog circuits, e.g., drift, offset, and sensitivity to temperature change, we used digital signal processing (DSP) and closed loop feedback to control the magnet field in the vacuum chamber. A prototype of the storage ring sextupole magnet was used in this work, which has geometry similar to that of the six-pole horizontal/vertical corrector magnet. The effect of the eddy current in the $0.025^{\prime \prime}$-thick magnet laminations was measured and found to be negligibly small in a previous work. [1]

This paper will be largely divided into two parts. In the first part, we will overview Z-transform and theory of digital signal processing and in the second part, we will present measurement of eddy current effect and its compensation using a digital filter and closed loop feedback. $[2,3]$

\section{DIGITAL SIGNAL PROCESSING}

In this section, theory of closed loop feedback using digital signal processing based on Z-transform [2] will be discussed.

\section{A. Digital Filters}

Given a discreet input sequence $\left\{\mathrm{x}_{\mathrm{n}}\right\}$ sampled at every $\mathrm{T}(=$ $\left.1 / F_{s}\right)$, a digital filter produces an output sequence $\left\{y_{n}\right\}$ given by the difference equation

$$
y_{n}=\sum_{k=0}^{M} a_{k} x_{n-k}-\sum_{k=1}^{L} b_{k} y_{n-k}
$$

where $\left\{a_{k}\right\}$ and $\left\{b_{k}\right\}$ are the filter coefficients. $F_{s}$ is called the sampling frequency. The filter takes $M$ previous inputs plus the current one and $L$ previous outputs, and therefore, the filter is physically realizable or causal. Performing a Ztransform on Eq. (1), we obtain

$$
\mathrm{Y}(\mathrm{z})=\mathrm{H}(\mathrm{z}) \mathrm{X}(\mathrm{z})
$$

where $X(z)$ and $Y(z)$ are the $Z$-transforms of $\left\{x_{n}\right\}$ and $\left\{y_{n}\right\}$ and $H(z)$ is the filter function given by

$$
H(z)=\frac{\sum_{k=0}^{M} a_{k} z^{-k}}{1+\sum_{k=1}^{L} b_{k} z^{-k}} .
$$

With $\mathrm{z}=\mathrm{e}^{-\mathrm{i} \lambda}$ and $\lambda=\pi \mathrm{f} / \mathrm{F}_{\mathrm{s}}$, the $\mathrm{Z}$-transform is the digital analog of the Fourier transform.

The inverse of the filter H(z) in Eq. (3) can be written as

$$
\frac{1}{H(z)}=\frac{1}{a_{0}} \frac{\sum_{k=0}^{L} b_{k} z^{-k}}{1+\sum_{k=1}^{M} \frac{a_{k}}{a_{0}} z^{-k}} \cdot \quad\left(b_{0}=1\right)
$$

\footnotetext{
*Work supported by the U.S. Department of Energy, Office of
} Basic Energy Sciences, under Contract No. W-31-109-ENG-38. 
The inverse filter in Eq. (4) can be used to compensate for analog devices if the corresponding filter coefficients $\left\{a_{k}\right\}$ and $\left\{b_{k}\right\}$ can be obtained by fitting the frequency response to Eq. (3) with $z=e^{-i \lambda}$. We will use this approach to compensate for the effect of eddy current in the vacuum chamber.

\section{B. Closed Loop Feedback}

In Fig. 1 is shown a simple closed loop feedback system with proportional control of open loop gain G. The bandlimiting filter (BLF) is inserted in the loop for stability reasons. It is a low-pass filter with bandwidth $f_{b}$ and has the filter function

$$
B(z)=\frac{1+z^{-1}}{1+c+(1-c) z^{-1}} . \quad c=\cot \left(\frac{\pi f_{b}}{F_{s}}\right)
$$

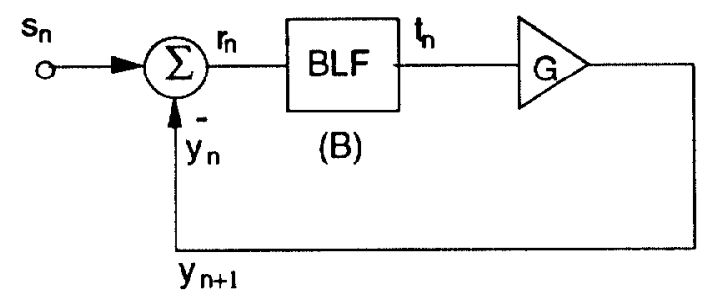

Fig. 1: Closed loop feedback with digital signal processing. $B L F$ is the bandlimiting filter with bandwidth of $\mathrm{f}_{\mathrm{b}}$.

The response of the system in the form of a difference equation is given by

$$
(1+c) y_{n+1}+(1-c+G) y_{n}+G y_{n-1}=G\left(s_{n}+s_{n-1}\right)
$$

Assuming $F_{s} \gg f_{b}$, Eq. (6) can be converted to a second-order differential equation and Fourier analyzed. In general, a closed loop feedback system with $\mathbf{k}$ open loop poles can be represented as a filter of $\mathrm{k}+1$ poles in the complex plante.

The system is stable if $G<1+c$ and, assuming $G \gg 1$, the bandwidth of the closed loop system is approximately equal to $\mathrm{G}_{\mathrm{b}}$. It can be shown that the condition for critically damped response is

$$
\frac{F_{s}}{G f_{b}} \approx 20,
$$

and therefore, the bandwidth of the optimally controlled feedback system is roughly equal to $F_{s} / 20$.

\section{MEASUREMENTS}

In this section, we will present results of measurements of the effect of the eddy current in the vacuum chamber and its compensation using digital filters.

\section{A. Magnet Impedance}

The magnet resistance $R$ and inductance $L$ can be obtained by measuring the ratio of voltage and current on the magnet as a function of frequency. The results show large changes both in $R$ and L. For the horizontal field, they changed from 100 $\mathrm{m} \Omega$ and $11.8 \mathrm{mH}$ at $\mathrm{DC}$ to $920 \mathrm{~m} \Omega$ and $8.2 \mathrm{mH}$ at $200 \mathrm{~Hz}$. For the vertical field, the changes were from $80 \mathrm{~m} \Omega$ and 8.7 $\mathrm{mH}$ at $\mathrm{DC}$ to $460 \mathrm{~m} \Omega$ and $4.9 \mathrm{mH}$ at $200 \mathrm{~Hz}$. These are mainly attributable to the eddy current in the vacuum chamber. While this has the effect of reducing the magnet time constant, it also increases power consumption.

\section{B. Field Attenuation and Phase Shift}

In Figs. 2(a) and 2(b) are shown the attenuation and phase shift of the field relative to the magnet current as functions of frequency at the center of the vacuum chamber $(x=0)$. The vertical field shows stronger attenuation and phase shift with frequency than the horizontal field, though the horizontal field appears to catch up in the high frequency region.

Spatially resolved measurement of the vertical magnet field in the vacuum chamber shows significant quadrupole and sextupole components. Below $20 \mathrm{~Hz}$, the quadrupole component dominates $\left(\left|b_{1}\right| \approx 0.12 \mathrm{~cm}^{-1} @ 20 \mathrm{~Hz}\right)$, while above $50 \mathrm{~Hz}$ the sextupole component becomes stronger $\left(\left|b_{2}\right| \approx 0.16\right.$ $\mathrm{cm}^{-2} @ 100 \mathrm{~Hz}$ ). This will cause bump closure error in the local feedback systems and interference among them as a result. The resolution of this effect will be done by the global feedback system.
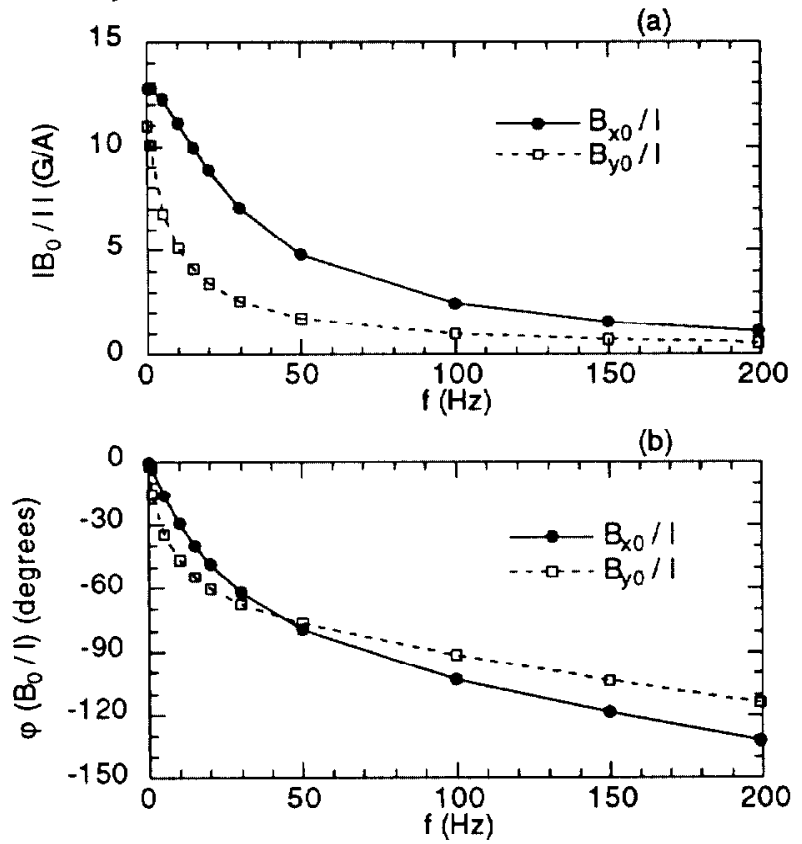

Fig. 2: (a) Attenuation of the field efficiency $\mid B_{x, y 0} / I$ and (b) phase shift $\varphi$ between the field and the current as functions of frequency at the center of the vacuum chamber $(x=0)$.

\section{Closed Loop Feedback with PID Control}

Figure 3 shows the schematic diagram for closed loop feedback with PID control for the magnet field in the vacuum chamber. A bandlimiting filter (BLF) of $30 \mathrm{~Hz}$ was used for these measurements. The analog reconstruction filter (ARF) is an analog low pass filter of $200 \mathrm{~Hz}$ bandwidth inserted in front of the power supply control input in order to eliminate 


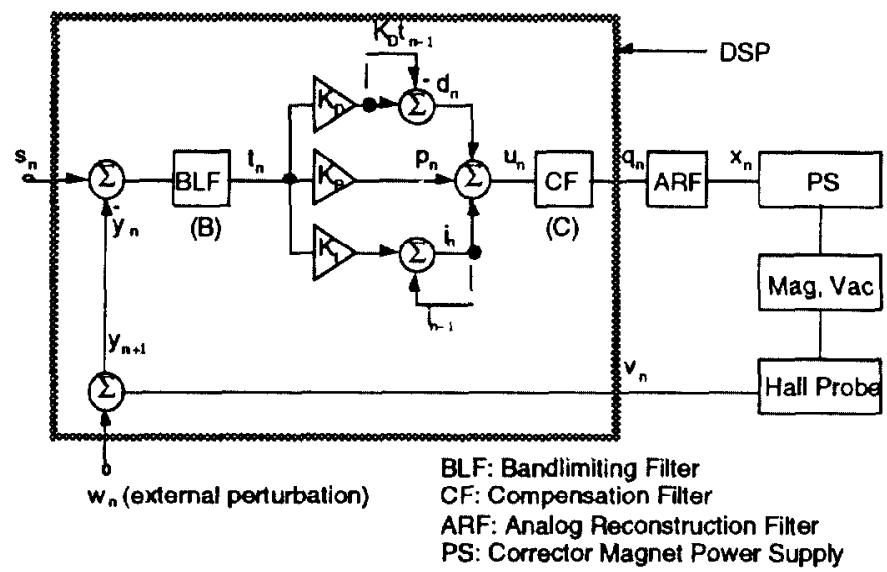

Fig. 3: Schematic diagram for the closed loop feedback with PID control.
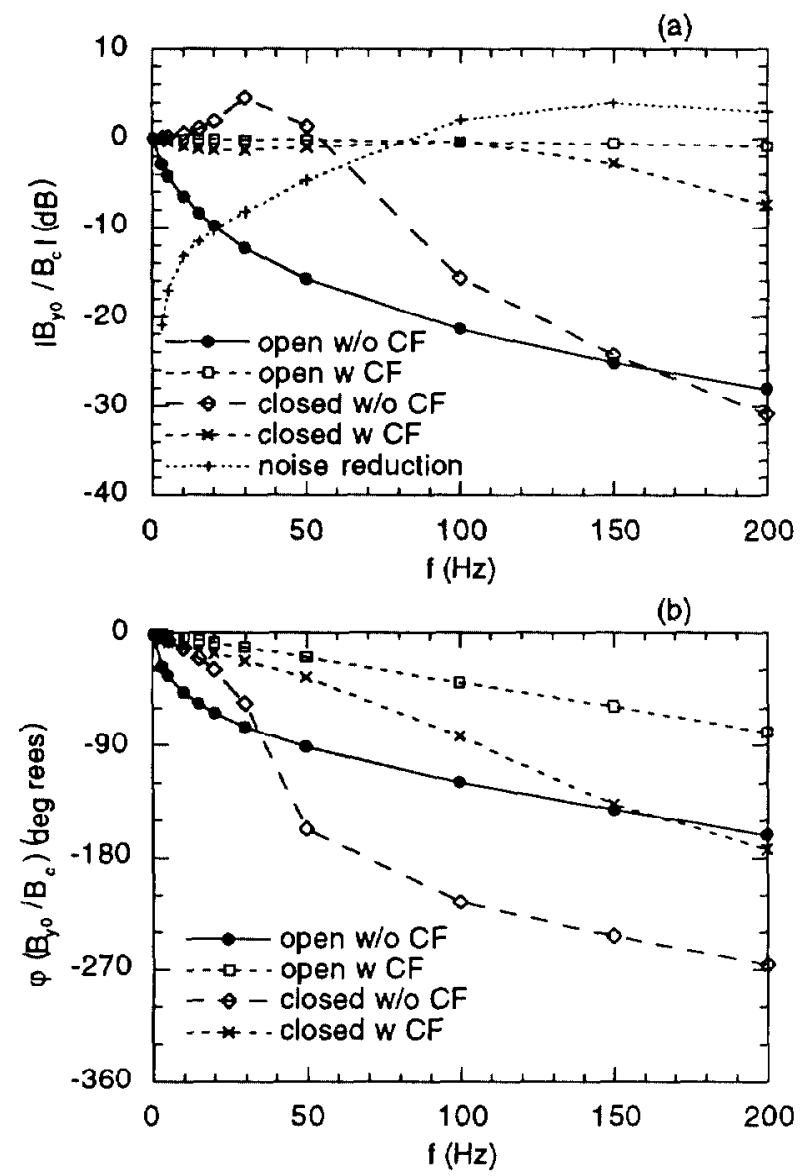

Fig. 4: Results of measurement on the closed loop feedback for corrector magnet field: (a) amplitude attenuation and (b) phase shift. The parameters used were: $F_{s}=4 \mathrm{kHz}, f_{b}=30$ $\mathrm{Hz}, \mathrm{K}_{\mathrm{P}}=3, \mathrm{~K}_{\mathrm{I}}=0.05$, and $\mathrm{K}_{\mathrm{D}}=0.5$.

high frequency components in the output from DSP. It was also intended to simulate the BPM, which will have finite bandwidth due to averaging over many turns. ARF was used only for closed loop measurements.

For the open loop measurements of the frequency response, the control signal is at either the input of the CF $\left(u_{n}\right)$ or the PS $\left(x_{n}\right)$ in Fig. 3. For the closed loop measurements, the control signal is at the input of the DSP $\left(s_{n}\right)$.

The digital compensation filter (CF) was obtained by inverting the digital filter representation of the vacuum chamber. Using the measurement results shown in Fig. 2(a), the best fit with four poles and four zeroes gives

$$
\begin{gathered}
\mathrm{q}_{\mathrm{n}}=57.8\left(\mathrm{u}_{\mathrm{n}}-3.1575996823 \mathrm{u}_{\mathrm{n}-1}+3.5206625349 \mathrm{u}_{\mathrm{n}-2}-\right. \\
\left.1.56793656962 \mathrm{u}_{\mathrm{n}-3}+0.2048748912 \mathrm{u}_{\mathrm{n}-4}\right)+ \\
1.60395604698 \mathrm{q}_{\mathrm{n}-1}-0.276518570415 \mathrm{q}_{\mathrm{n}-2}- \\
0.30381547655 \mathrm{q}_{\mathrm{n}-3}-0.023772023183 \mathrm{q}_{\mathrm{n}-4} .
\end{gathered}
$$

Since no future samples from either the input or output are needed to calculate the current output, the filter is physically realizable or causal.

The results of measurements on $\mathrm{B}_{\mathrm{y} 0} / \mathrm{B}_{\mathrm{c}}$ ( $\mathrm{B}_{\mathrm{c}}$ : control signal) with and without $\mathrm{CF}$ is shown in Fig. 4. Figure 5 shows time domain measurements of the magnet field $B$ inside the vacuum chamber in response to the input square pulses of 15 $\mathrm{Hz}$ with the $\mathrm{CF}$ turned off and on.
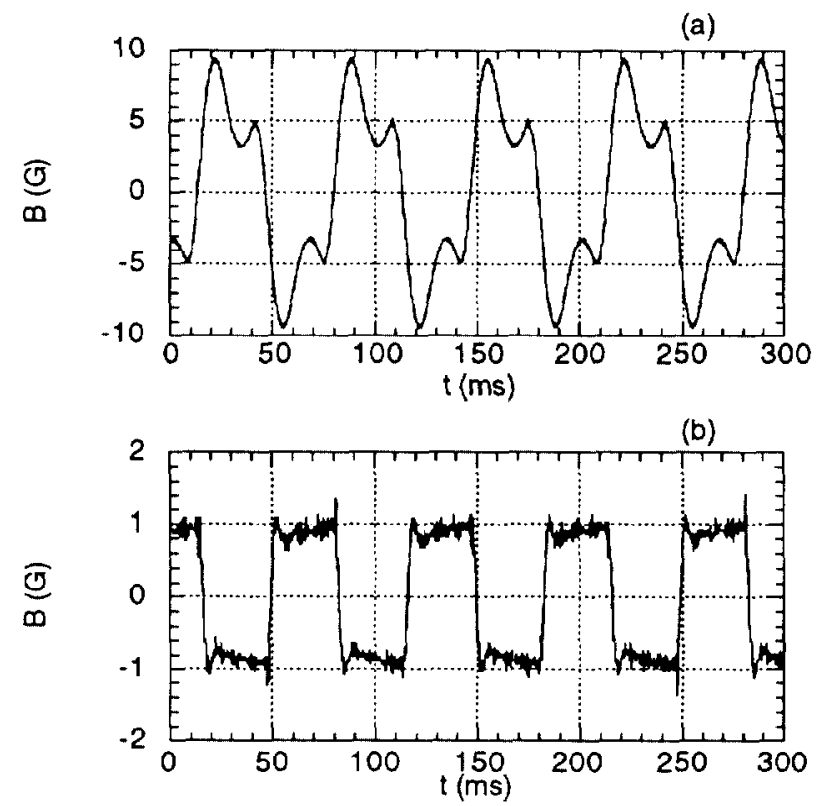

Fig. 5: Feedback system response in the time domain (a) without and (b) with eddy current compensation. The parameters used were: $F_{s}=4 \mathrm{kHz}, \mathrm{f}_{\mathrm{b}}=30 \mathrm{~Hz}, \mathrm{~K}_{\mathrm{P}}=3, \mathrm{~K}_{1}=$ 0.05 , and $K_{D}=0.5$. The control signal is a $15 \mathrm{~Hz}$ square wave.

\section{REFERENCES}

[1] Y. Chung and J. Galayda, "Effect of Eddy Current in the Laminations on the Magnet Field," LS Note 200, ANL, 1992.

[2] A. Peled and B. Liu, Digital Signal Processing, John Wiley \& Sons, 1976.

[3] Y. Chung, L. Emery, and K. Kirchman, "Digital Signal Processing for Beam Position Feedback," LS Note 202, ANL, 1992. 